\title{
Controle Ótimo em Agroecossistemas usando $\mathrm{SDRE}^{1}$
}

A. MOLTER ${ }^{2}$, Universidade Federal de Pelotas, Departamento de Matemática e Estatística, Campus Universitário, s/nº , Caixa Postal 354, 96010-900 Pelotas, RS, Brasil.

M. RAFIKOV ${ }^{3}$, Universidade Federal do ABC, Rua Santa Adélia, Centro de Engenharia, Modelagem e Ciências Sociais, 166, 09210-170 Santo André, SP, Brasil.

Resumo. O propósito deste trabalho é encontrar estratégias ótimas de controle de pragas no sistema biológico que apresenta comportamento não-linear. O controle, baseado no modelo de Lotka - Volterra de duas presas e um predador, é aplicado em um agroecossistema de plantação de soja. O objetivo desta estratégia de controle é manter a população de pragas abaixo de nível de danos econômicos. Este problema do controle ótimo é resolvido através do método das Equações de Riccati Dependentes do Estado (SDRE). Simulações numéricas para as estratégias do controle de pragas propostas, baseadas no modelo de Lotka-Volterra, foram realizadas para mostrar eficácia deste método.

Palavras-chave. Controle ótimo, sistemas biológicos, SDRE.

\section{Introdução}

Sistemas não lineares, exibindo comportamento caótico, tem despertado um crescente interesse dos pesquisadores. Os sistemas populacionais, incluindo sistemas presa - predador geralmente são não-lineares, e por vezes apresentam regime caótico $[6,13,14]$. Um modelo biológico deste tipo, de duas presas e um predador é o de Lotka-Volterra.

O controle biológico consiste em inserir predadores em meio as pragas que afetam as culturas ou causam algum mal à saúde humana ou aos animais. A grande vantagem desse controle é reduzir a quantidades toleráveis de pragas, sem causar danos ao meio ambiente ou a nossa saúde. Assim é possível chegar a um nível das espécias de maneira a manter o equilíbrio ambiental e econômico [10].

Dentre as diversas técnicas de controle de sistemas biológicos não-lineares [4, 8, 10, 11], o método baseado na solução das equações de Riccati Dependentes do

\footnotetext{
${ }^{1}$ Agradecemos ao apoio da FAPERGS, projeto 10/0091-3, ao apoio do CNPq e da FAPESP.

2alexandre.molter@yahoo.com.br

${ }^{3}$ marat9119@yahoo.com.br
} 
Estado é recente, surgiu a pouco mais de uma década [7] e tem contribuído para controlar sistemas altamente não-lineares, mostrando eficiência no controle $[2,3,12]$.

O objetivo desse trabalho é usar o método SDRE como uma estratégia de controle de sistemas biológicos que apresentam comportamento caótico e aplicar num agroecossistema (no plantio da soja do Noroeste do estado do Rio Grande do Sul), que inclui espécies de duas pragas, encontradas nos municípios da região Noroeste do estado do Rio Grande do Sul: Rachiplusia nu e Pseudoplusia includes (lagartas da soja) e um predador ou parasitóide dessas duas espécies.

Rachiplusia nu - ocorre na maioria dos estados produtores de soja do Brasil, além disso, é amplamente distribuída pela América do Sul e América do Norte. Alimenta-se de alfafa, funcho, girassol, linho, rabanete, repolho, soja, fumo e outras plantas [1].

Pseudoplusia includes - é a mais importante da subfamília Plusia que ocorre em soja. Sua ocorrência predomina em São Paulo, Paraná e Rio Grande do Sul. Alimenta-se de folhagens [5].

Como é visto da Tabela 1, as características principais do ciclo biológico destas pragas são praticamente iguais.

Tabela 1: Ciclo Biológico (em dias) das espécies de presas.

\begin{tabular}{|c|c|c|}
\hline Ciclo biológico & $R . n u$ & $P$. includes \\
\hline Incubação dos ovos & 3,0 & 3,0 \\
\hline Período larval & 13,3 & 12,9 \\
\hline Período pupal & 7,0 & 7,0 \\
\hline Longevidade dos adultos & 11,5 & 12,0 \\
\hline Média de ovos por fêmea & 400 a 600 & 400 a 600 \\
\hline N $^{\circ}$ de gerações por safra & 3 a 4 & 3 a 4 \\
\hline
\end{tabular}

Fonte: Nakono et all (1981).

No trabalho assumiu-se a densidade aceitável de pragas abaixo de danos econômicos e ecológicos (ponto de equilíbrio de pragas), fornecidos pela EMBRAPA, como meta de controle de pragas. O ponto de equilíbrio do predador foi calculado a partir do ponto de equilíbrio natural do sistema, levando em conta os dados fornecidos pela EMBRAPA que recomenda o controle quando forem encontrados em média a densidade de 20 lagartas grandes por metro quadrado (com mais de 1,5 cm de comprimento) ou 40 lagartas pequenas (de 0,5 a $1,5 \mathrm{~cm}$ de comprimento), por amostragem. Este número foi obtido a partir de cálculos envolvendo a área foliar da cultura, sua capacidade de recuperação e a capacidade de consumo das lagartas. Para simulações numéricas do sistema foi usado o software MATLAB. 


\section{Modelo Matemático do Sistema Duas Presas - Um Predador}

O modelo de Lotka-Volterra com duas presas e um predador tem a seguinte forma:

$$
\frac{d x_{i}}{d t}=x_{i}\left(r_{i}-\sum_{j=1}^{3} a_{i j} x_{j}\right), \quad i=1,2,3,
$$

em que, $x_{1}, x_{2}$ e $x_{3}$ são a densidade da primeira presa, segunda presa e predador respectivamente. Os coeficientes $r_{1}$ e $r_{2}$ caracterizam a taxa de reprodução das duas espécies de pragas em consideração; o coeficiente $r_{3}$ tem sinal negativo e caracteriza a taxa de mortalidade natural de predador. Os coeficientes e $a_{i j}, i, j=1,2,3$ caracterizam taxas de interações entre espécies do sistema.

Para determinação da taxa de reprodução das espécies $R$. nu e P. includes, será considerada uma situação de laboratório, com desenvolvimento livre, sem valor de saturação, ou seja, se considera o modelo de Malthus: $\frac{d N}{d t}=r_{1} N$, onde $r_{1}$ é a taxa de crescimento da População $N$.

No caso da $R$. nu o tempo de vida é em torno de 35 dias, considerando o ciclo biológico completo, desde a incubação até o final da fase adulta, conforme apresentado na Tabela 1. Aqui não será levada em conta que há uma queda na fertilidade quando se encontra no fim da fase adulta. Como média de ovos por fêmea usa-se 400. Pelo modelo de Malthus tem-se:

$N=N_{0} e^{r_{1} t}$, usando os dados da tabela, encontra-se

$400=1 . e^{35 r_{1}}$, isso implica em

$\ln (400)=35 r_{1}$, daí vem que

$r_{1}=\ln (400) / 35=0,17$.

Considerando que o tempo da vida e média de ovos para fêmea das pragas são praticamente iguais, concluímos que $r_{2}=0,17$.

Supondo que as duas pragas têm a mesma capacidade de suporte $K=100$, calculamos valores os de $a_{11}=a_{22}=0,0017$. Foram assumidos os seguintes coeficientes de captura $a_{13}=a_{23}=0,0017$ e os valores de coeficientes de competição $a_{12}=0,00017$ e $a_{21}=0,000255$ [10].

A expectativa de vida do predador ou parasitóide é considerada 5,9 dias, então o coeficiente de mortalidade $r_{3}=-1 / 5,9=-0,17$. De acordo com [10], os coeficientes de conversão são $a_{31}=-0,00085$ e $a_{32}=-0,000085$. Como é visto, a influência da segunda presa na reprodução do predador é 10 vezes menor que a da primeira. Esta situação pode acontecer na realidade se a segunda praga for contaminada por uma doença específica dela. Espécies parasitadas e contaminadas de pragas não deixam finalizar o ciclo de desenvolvimento do parasitóide. Atribuindo os valores acima referidos aos coeficientes do sistema (2.1), obtemos

$$
\begin{array}{r}
\frac{d x_{1}}{d t}=x_{1}\left(0,17-0,0017 x_{1}-0,00017 x_{2}-0,0017 x_{3}\right) \\
\frac{d x_{2}}{d t}=x_{2}\left(0,17-0,000255 x_{1}-0,0017 x_{2}-0,0017 x_{3}\right) \\
\quad \frac{d x_{3}}{d t}=x_{3}\left(-0,17+0,00085 x_{1}+0,000085 x_{2}\right) .
\end{array}
$$


O sistema não-linear (2.2) apresenta comportamento caótico, como mostra a Figura 1, para condições iniciais $(20,30,1)$.
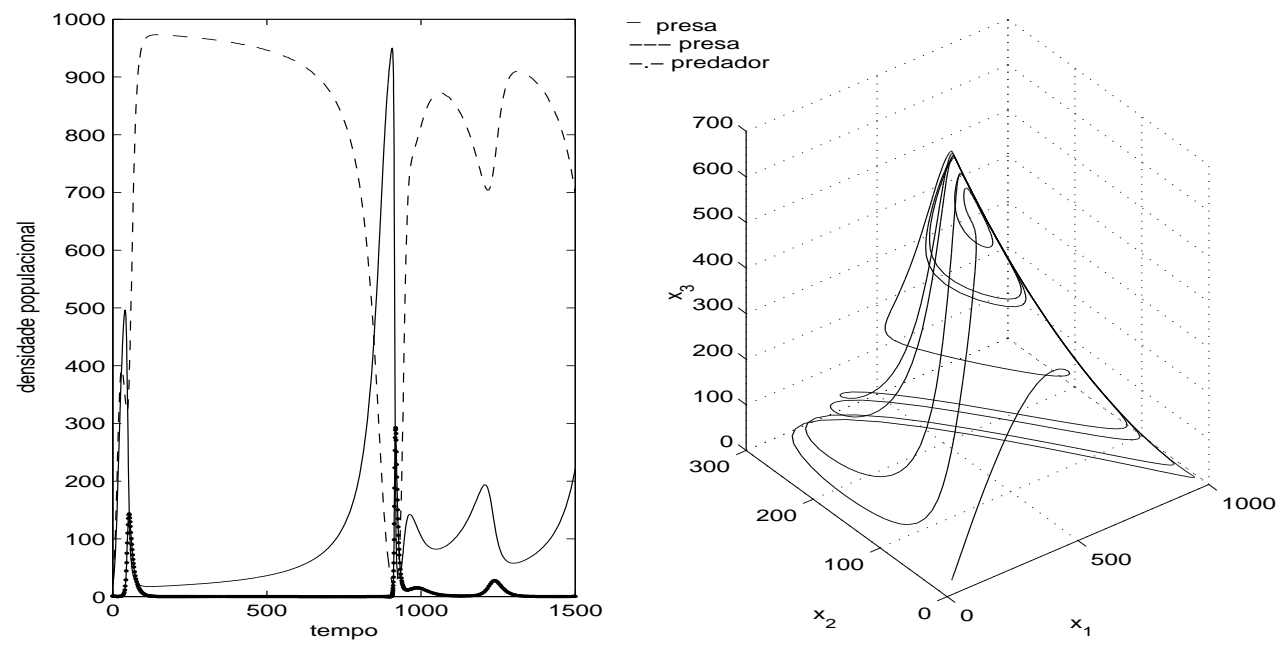

Figura 1: Trajetórias do sistema com parâmetros reais e diagrama de fase.

O ponto de equilíbrio natural $\left(\bar{x}_{1}, \bar{x}_{2}, \bar{x}_{3}\right)$ do modelo é calculado do sistema

$$
\begin{array}{r}
1-0,001 \bar{x}_{1}-0,001 \bar{x}_{2}-0,01 \bar{x}_{3}=0 \\
1-0,0015 \bar{x}_{1}-0,001 \bar{x}_{2}-0,001 \bar{x}_{3}=0 \\
-1+0,005 \bar{x}_{1}+0,0005 \bar{x}_{2}=0,
\end{array}
$$

cujo valor é $\left(\bar{x}_{1}, \bar{x}_{2}, \bar{x}_{3}\right)=(118,4211 ; 815,7895 ; 6,5789)$. É possível verificar que esse é um ponto de equilíbrio instável.

\section{Controle Ótimo com Realimentação para Siste- mas Não-Lineares por SDRE}

Um problema de controle ótimo para um sistema com os coeficientes das matrizes de estado, dependentes do estado e horizonte infinito, pode ser formulado da seguinte forma [7]: minimizar o funcional de custo

$$
J\left(\mathbf{x}_{0}, \mathbf{u}\right)=\frac{1}{2} \int_{t_{0}}^{\infty}\left(\mathbf{x}^{T} \mathbf{Q}(x) \mathbf{x}+\mathbf{u}^{T} \mathbf{R}(x) \mathbf{u}\right) d t
$$

em relação ao estado $\mathbf{x}$ e o controle $\mathbf{u}$, sujeito ao sistema de restrições não-lineares

$$
\begin{aligned}
\dot{\mathbf{x}} & =\mathbf{f}(x)+\mathbf{B}(x) \mathbf{u}, \\
\mathbf{z} & =\mathbf{C}(x) \mathbf{x}, \\
\mathbf{x}(0) & =\mathbf{x}_{0}
\end{aligned}
$$


onde $\mathbf{B} \in \mathbf{R}^{n \times m}$ é a matriz de controle, $\mathbf{C}$ é a matriz de saída do sistema, $\mathbf{x} \in \mathbf{R}^{n}$, $\mathbf{u} \in \mathbf{R}^{m}$ e $\mathbf{z} \in \mathbf{R}^{s}$ ( $\mathbf{R}^{s}$ é a dimensão do vetor de saída do sistema). $\mathbf{x}(0)$ é a condição inicial, $\mathbf{Q}(x) \in \mathbf{R}^{n \times n}$ é matriz de pesos semidefinida positiva e $\mathbf{R}(x) \in \mathbf{R}^{m \times m}$ é definida positiva.

A aproximação pelas equações de Riccati dependentes do estado, para resolver o problema de controle subótimo (3.1) e (3.2) é dada pelas seguintes etapas:

1- Usar parametrização direta para transformar a dinâmica não-linear do estado em matrizes de coeficientes dependentes do estado (SDC).

As equações do sistema dinâmico com controle são dadas por

$$
\dot{\mathbf{x}}=\mathbf{A}(x) \mathbf{x}+\mathbf{B}(x) \mathbf{u},
$$

com $\mathbf{f}(x)=\mathbf{A}(x) \mathbf{x}$ sendo $\mathbf{A} \in \mathbf{R}^{n \times n}$ a matriz de estado. Em geral, $\mathbf{A}(x)$ é unica somente se $x$ for escalar [2].

De fato há um número infinito de parametizações para os coeficientes dependentes do estado. Isto é vardadeiro desde que existam ao menos duas parametrizaçãos para todo $0 \leq \alpha \leq 1$ satisfazendo

$$
\alpha A_{1}(x) x+(1-\alpha) A_{2}(x) x=\alpha f(x)+(1-\alpha) f(x)=f(x) .
$$

A escolha das parametrizações a serem feitas deve ser apropriada, de acordo com o sistema de controle de interesse. Um fator importante para esta escolha é não violar a controlabilidade do sistema, ou seja, a matriz de controlabilidade dependente do estado $\left[\begin{array}{llll}\mathbf{B}(x) & \mathbf{A}(x) \mathbf{B}(x) & \ldots & \mathbf{A}^{n-1}(x) \mathbf{B}(x)\end{array}\right]$ ter posto completo.

2- Resolver a equação de Riccati dependente do estado [2].

As condições necessárias para otimalidade podem ser obtidas usando o cálculo variacional. O hamiltoniano para o problema de controle ótimo (3.1) e (3.2) é dado por

$$
\mathbf{H}(\mathbf{x}, \mathbf{u}, \lambda)=\frac{1}{2}\left(\mathbf{x}^{T} \mathbf{Q}(x) \mathbf{x}+\mathbf{u}^{T} \mathbf{R}(x) \mathbf{u}\right)+\lambda^{T}(\mathbf{A}(x) \mathbf{x}+\mathbf{B}(x) \mathbf{u}) .
$$

onde $\lambda \in \mathbf{R}^{n}$ é o multiplicador de Lagrange. As condições necessárias para o controle ótimo são $\dot{\mathbf{x}}=\partial \mathbf{H} / \partial \lambda, \partial \mathbf{H} / \partial \mathbf{u}=0$ e $\dot{\lambda}=-\partial \mathbf{H} / \partial \mathbf{x}$. Do hamiltoniano obtem-se

$$
\begin{aligned}
\dot{\lambda}=-\mathbf{Q}(x) \mathbf{x}-\frac{1}{2} \mathbf{x}^{T} \frac{\partial \mathbf{Q}(x)}{\partial x} \mathbf{x}-\frac{1}{2} \mathbf{u}^{T} \frac{\partial \mathbf{R}(x)}{\partial x} \mathbf{u}-\left[\frac{\partial(\mathbf{A}(x) \mathbf{x})}{\partial x}\right]^{T} \lambda-\left[\frac{\partial(\mathbf{B}(x) \mathbf{u})}{\partial x}\right]^{T} \lambda, \\
\dot{\mathbf{x}}=\mathbf{A}(x) \mathbf{x}+\mathbf{B}(x) \mathbf{u}, \\
0=\mathbf{R}(x) \mathbf{u}+\mathbf{B}(x) \lambda .
\end{aligned}
$$

Denotando $\mathbf{A}_{i}$ a $i$-linha de $\mathbf{A}(x)$ e $\mathbf{B}_{i}$ a $i$-linha de $\mathbf{B}(x)$. Como pode ser visto em [2], os termos de derivada parcial, na forma matricial são

$$
\frac{\partial(\mathbf{A}(x) \mathbf{x})}{\partial x}=\mathbf{A}(x)+\frac{\partial(\mathbf{A}(x))}{\partial x} \mathbf{x}=\mathbf{A}(x)+\left[\begin{array}{ccc}
\frac{\partial \mathbf{A}_{1}}{\partial x_{1}} \mathbf{x} & \cdots & \frac{\partial \mathbf{A}_{1}}{\partial x_{n}} \mathbf{x} \\
\cdots & \ddots & \cdots \\
\frac{\partial \mathbf{A}_{n}}{\partial x_{1}} \mathbf{x} & \cdots & \frac{\partial \mathbf{A}_{n}}{\partial x_{n}} \mathbf{x}
\end{array}\right],
$$


$\mathrm{e}$

$$
\frac{\partial(\mathbf{B}(x) \mathbf{u})}{\partial x}=\left[\begin{array}{ccc}
\frac{\partial \mathbf{B}_{1}}{\partial x_{1}} \mathbf{u} & \cdots & \frac{\partial \mathbf{B}_{1}}{\partial x_{n}} \mathbf{u} \\
\cdots & \ddots & \cdots \\
\frac{\partial \mathbf{B}_{n}}{\partial x_{1}} \mathbf{u} & \cdots & \frac{\partial \mathbf{B}_{n}}{\partial x_{n}} \mathbf{u}
\end{array}\right]
$$

O co-estado é assumido na forma $\lambda=\mathbf{P}(x) \mathbf{x}$, que tem dependência do estado. Usando esta forma do co-estado, da equação (3.8) obtem-se o controle realimentado

$$
\mathbf{u}=-\mathbf{R}^{-1}(x) \mathbf{B}^{T}(x) \mathbf{P}(x) \mathbf{x} .
$$

Substituindo este controle na equação (3.7) tem-se

$$
\dot{\mathbf{x}}=\mathbf{A}(x) \mathbf{x}-\mathbf{B}(x) \mathbf{R}^{-1}(x) \mathbf{B}^{T}(x) \mathbf{P}(x) \mathbf{x} .
$$

Para encontrar o valor da função $\mathbf{P}(x)$ diferencia-se $\lambda=\mathbf{P}(x) \mathbf{x}$ no tempo ao longo da trajetória

$$
\begin{array}{r}
\dot{\lambda}=\dot{\mathbf{P}}(x) \mathbf{x}+\mathbf{P}(x) \dot{\mathbf{x}} \\
=\dot{\mathbf{P}}(x) \mathbf{x}+\mathbf{P}(x) \mathbf{A}(x) \mathbf{x}-\mathbf{P}(x) \mathbf{B}(x) \mathbf{R}^{-1}(x) \mathbf{B}^{T}(x) \mathbf{P}(x) \mathbf{x},
\end{array}
$$

onde é usada a notação $\dot{\mathbf{P}}(x)=\sum_{i=1}^{n} P_{x_{i}}(x) \dot{x}_{i}(t)$.

Substituindo a equação (3.13) na primeira condição necessária do controle ótimo, equação (3.6), referente à $\dot{\lambda}$, obtem-se

$$
\begin{array}{r}
\dot{\mathbf{P}}(x) \mathbf{x}+\mathbf{P}(x) \mathbf{A}(x) \mathbf{x}-\mathbf{P}(x) \mathbf{B}(x) \mathbf{R}^{-1}(x) \mathbf{B}^{T}(x) \mathbf{P}(x) \mathbf{x} \\
=-\mathbf{Q}(x) \mathbf{x}-\frac{1}{2} \mathbf{x}^{T} \frac{\partial \mathbf{Q}(x)}{\partial x} \mathbf{x}-\frac{1}{2} \mathbf{u}^{T} \frac{\partial \mathbf{R}(x)}{\partial x} \mathbf{u} \\
-\left[\mathbf{A}(x)+\frac{\partial(\mathbf{A}(x))}{\partial x} \mathbf{x}\right]^{T} \mathbf{P}(x) \mathbf{x}-\left[\frac{\partial(\mathbf{B}(x) \mathbf{u})}{\partial x}\right]^{T} \mathbf{P}(x) \mathbf{x},
\end{array}
$$

Colocando os termos de uma maneira mais adequada, tem-se

$$
\begin{array}{r}
\dot{\mathbf{P}}(x) \mathbf{x}+\frac{1}{2} \mathbf{x}^{T} \frac{\partial \mathbf{Q}(x)}{\partial x} \mathbf{x}+\frac{1}{2} \mathbf{u}^{T} \frac{\partial \mathbf{R}(x)}{\partial x} \mathbf{u}+ \\
\mathbf{x}^{T}\left[\frac{\partial(\mathbf{A}(x))}{\partial x}\right]^{T} \mathbf{P}(x) \mathbf{x}+\left[\frac{\partial(\mathbf{B}(x) \mathbf{u})}{\partial x}\right]^{T} \mathbf{P}(x) \mathbf{x}+ \\
{\left[\mathbf{P}(x) \mathbf{A}(x)+\mathbf{A}^{T}(x) \mathbf{P}(x)-\mathbf{P}(x) \mathbf{B}(x) \mathbf{R}^{-1}(x) \mathbf{B}^{T}(x) \mathbf{P}(x)+\mathbf{Q}(x)\right] \mathbf{x}=0,}
\end{array}
$$

Assumindo que $\mathbf{P}(x)$ é solução da equação de Riccati dependente do estado (SDRE), dada por

$$
\mathbf{P}(x) \mathbf{A}(x)+\mathbf{A}^{T}(x) \mathbf{P}(x)-\mathbf{P}(x) \mathbf{B}(x) \mathbf{R}^{-1}(x) \mathbf{B}^{T}(x) \mathbf{P}(x)+\mathbf{Q}(x)=0,
$$

então a seguinte condição necessária de otimalidade precisa ser satisfeita

$$
\begin{array}{r}
\dot{\mathbf{P}}(x) \mathbf{x}+\frac{1}{2} \mathbf{x}^{T} \frac{\partial \mathbf{Q}(x)}{\partial x} \mathbf{x}+\frac{1}{2} \mathbf{u}^{T} \frac{\partial \mathbf{R}(x)}{\partial x} \mathbf{u}+ \\
\mathbf{x}^{T}\left[\frac{\partial(\mathbf{A}(x))}{\partial x}\right]^{T} \mathbf{P}(x) \mathbf{x}+\left[\frac{\partial(\mathbf{B}(x) \mathbf{u})}{\partial x}\right]^{T} \mathbf{P}(x) \mathbf{x}=0 .
\end{array}
$$


No tempo infinito, no caso padrão do Regulador Linear Quadrático, esta é uma condição de otimalidade que satisfaz a solução do controle subótimo localmente.

3 - Construir o controle não-linear realimentado [2]

$$
\begin{array}{r}
\mathbf{u}=-\mathbf{S}(x) \mathbf{x}, \\
\mathbf{S}(x)=\mathbf{R}^{-1}(x) \mathbf{B}^{T}(x) \mathbf{P}(x) .
\end{array}
$$

Para alguns casos especiais, como sistemas com pouca dependência do estado ou com poucas variáveis de estado, a equação (3.16) pode ser resolvida de forma analítica [12]. Por outro lado, uma solução numérica pode ser obtida com uma taxa de amostragem suficientemente grande. Isto quer dizer que podemos resolver a equação de Riccati substituindo os valores $\mathbf{x}_{0}$ no sistema, resolver $\mathbf{P A}+\mathbf{A}^{T} \mathbf{P}-$ $\mathbf{P B R}^{-1} \mathbf{B}^{T} \mathbf{P}+\mathbf{Q}=0$ encontrando $\mathbf{P}, \mathbf{u}$ e um novo $\mathbf{x}$ e substituir novamente, e assim por diante até atingir o último passo no intervalo requerido. Isto é possível desde que o incremento de tempo seja suficientemte pequeno. Uma aproximação, com estabilidade local, do sistema de malha fechada é resultado do uso da técnica das equações de Riccati dependentes do estado, não-lineares.

Lema 3.1. Para uma parametrização dos coeficientes dependentes do estado $\mathbf{A}(x) \mathbf{x}$, $\mathbf{A}(0)$ é a linearização de $f(x)$ sobre o zero equilíbrio.

A demonstração deste Lema pode ser vista em [2].

É assumido que existe solução da SDRE para todo $\mathbf{x}$ na vizinhança da origem considerada. Então, naturalmente, o par $(\mathbf{A}(x), \mathbf{B}(x))$ é uma parametrização estabelizável. Uma consequência lógica é que a solução existe em $\mathbf{x}=0$ e que $\mathbf{P}_{0}=\mathbf{P}(0)$ é solução do sistema de equações algébricas de Riccati (3.16).

Teorema 3.1. [7] - Assume-se que a parametrização dos coeficientes dependentes do estado seja escolhida de forma que a coluna $\mathbf{A}(x) \in C^{1}$ em torno da vizinhança sobre a origem e que os pares $(\mathbf{A}(x), \mathbf{B}(x))$ e $(\mathbf{C}(x), \mathbf{A}(x))$ sejam, no sentido linear para todo $x$ pertencente a vizinhança sobre a origem, ponto a ponto, estabilizáveis e detectáveis, respectivamente. Então o regulador não-linear SDRE produz uma solução em malha fechada que é localmente e assintoticamente estável.

Este teorema é demonstrado em $[2,7]$.

Segundo [7], um fator importante do método SDRE é que ele não cancela os benefícios que podem provir das não-linearidades do sistema dinâmico. A razão para isto pode ser que ele não exige nenhuma inversão dinâmica e nem linearizações na realimentação do sistema não-linear.

\section{Simulações}

Nesta técnica de controle é considerada uma situação inicial $\mathbf{y}_{0}$ e procura-se levar o sistema a um ponto de equilíbrio desejado, situação final. A proposta é introduzir 
um número de predadores que mantenha o sistema naturalmente equilibrado, sem danos ao meio ambiente e ao cultivo da soja. O problema de controle subótimo (3.1) e (3.2), para soluções ótimas ponto a ponto, pode ser reformulado em função de um vetor com desvios $\mathbf{y}$ : minimizar o funcional de custo quadrático

$$
J_{y}=\frac{1}{2} \int_{0}^{\infty}\left(\mathbf{y}^{T} \mathbf{Q y}+\mathbf{u}^{T} \mathbf{R u}\right) d t
$$

em relação ao estado $\mathbf{y}$ e o controle $\mathbf{u}$, sujeito ao sistema de restrições não-lineares

$$
\begin{gathered}
\dot{\mathbf{y}}=\mathbf{A}(x) \mathbf{y}+\mathbf{B}(x) \mathbf{u}, \\
\mathbf{z}=\mathbf{C}(x) \mathbf{y}, \quad \mathbf{y}(0)=\mathbf{y}_{0}, \quad \mathbf{y}(\infty)=\mathbf{0} .
\end{gathered}
$$

Consideramos o sistema Lotka-Volterra (2.2) incluimos nele funções de controle $u=-s y$.

Em termos biológicos, a forma ideal para este sistema é controle escalar $U$, inserindo somente predadores no sistema da seguinte maneira:

$$
\begin{aligned}
& \dot{x}_{1}=x_{1}\left(r_{1}-a_{11} x_{1}-a_{12} x_{2}-a_{13} x_{3}\right) \\
& \dot{x}_{2}=x_{2}\left(r_{2}-a_{21} x_{1}-a_{22} x_{2}-a_{23} x_{3}\right) \\
& \dot{x}_{3}=x_{3}\left(r_{3}-a_{31} x_{1}-a_{32} x_{2}-a_{33} x_{3}\right)+U .
\end{aligned}
$$

Introduzimos os desvios

$$
\begin{aligned}
x_{1} & =y_{1}+x_{1}^{*} \\
x_{2} & =y_{2}+x_{2}^{*} \\
U & =u+u^{*},
\end{aligned}
$$

perturbando o sistema e buscando um controle que leve ao estado estacionário desejado

$$
\begin{aligned}
& x_{1}^{*}\left(r_{1}-a_{11} x_{1}^{*}-a_{12} x_{2}^{*}-a_{13} x_{3}^{*}\right)=0 \\
& x_{2}^{*}\left(r_{2}-a_{21} x_{1}^{*}-a_{22} x_{2}^{*}-a_{23} x_{3}^{*}\right)=0 \\
& x_{3}^{*}\left(r_{3}-a_{31} x_{1}^{*}-a_{32} x_{2}^{*}-a_{33} x_{3}^{*}\right)+u^{*}=0 .
\end{aligned}
$$

O número de pragas abaixo de danos econômicos é 20, portanto consideramos $x_{1}^{*}+x_{2}^{*}=18$. Para qualquer condição inicial, com este modelo não é possível assegurar o nível de pragas abaixo de danos econômicos $x_{1}^{*}+x_{2}^{*}=18$, como foi mostrado em [10], pois a quantidade de predadores introduzidos no sistema é insuficiente.

Neste caso, podemos introduzir um novo parasitóide, com intuito de estabilizar o sistema abaixo de danos econômicos. Este novo predador poderá ter características similares ao anterior. Consideremos um sistema com duas presas e dois predadores, introduzindo duas funções de controle $U_{1}$ e $U_{2}$, sendo modelado pelas seguintes equações diferenciais:

$$
\begin{aligned}
& \dot{x}_{1}=x_{1}\left(r_{1}-a_{11} x_{1}-a_{12} x_{2}-a_{13} x_{3}-a_{14} x_{4}\right) \\
& \dot{x}_{2}=x_{2}\left(r_{2}-a_{21} x_{1}-a_{22} x_{2}-a_{23} x_{3}-a_{24} x_{4}\right) \\
& \dot{x}_{3}=x_{3}\left(r_{3}-a_{31} x_{1}-a_{32} x_{2}-a_{33} x_{3}-a_{34} x_{4}\right)+U_{1} \\
& \dot{x}_{4}=x_{4}\left(r_{4}-a_{41} x_{1}-a_{42} x_{2}-a_{43} x_{3}-a_{44} x_{4}\right)+U_{2},
\end{aligned}
$$


com os parâmetros $r_{4}=-0,17, a_{14}=0,0017, a_{24}=0,0017, a_{41}=-0,00425$, $a_{42}=-0,00425$ e $a_{43}=a_{44}=0$ [10]. Introduzimos os desvios de forma similiar aos da equação (4.4). Substituindo os desvios (4.4) neste novo modelo (4.6) e admitindo o estado estacionário (4.5), temos

$$
\begin{aligned}
& \dot{y}_{1}=\left(y_{1}+x_{1}^{*}\right)\left(-a_{11} y_{1}-a_{12} y_{2}-a_{13} y_{3}-a_{14} y_{4}\right) \\
& \dot{y}_{2}=\left(y_{2}+x_{2}^{*}\right)\left(-a_{21} y_{1}-a_{22} y_{2}-a_{23} y_{3}-a_{24} y_{4}\right) \\
& \dot{y}_{3}=\left(y_{3}+x_{3}^{*}\right)\left(r_{3}-a_{31} y_{1}-a_{31} x_{1}^{*}-a_{32} y_{2}-a_{32} x_{2}^{*}\right)+u_{1}+u_{1}^{*} \\
& \dot{y}_{4}=\left(y_{4}+x_{4}^{*}\right)\left(r_{4}-a_{41} y_{1}-a_{41} x_{1}^{*}-a_{42} y_{2}-a_{42} x_{2}^{*}\right)+u_{2}+u_{2}^{*} .
\end{aligned}
$$

Então, da equação de estado (4.2), uma parametrização para a matriz de coeficientes dependentes do estado $\mathbf{A}(x)$ e a matriz $\mathbf{B}$ são dadas por

$$
\begin{gathered}
\mathbf{A}=\left[\begin{array}{cccc}
-a_{11}\left(y_{1}+x_{1}^{*}\right) & -a_{12}\left(y_{1}+x_{1}^{*}\right) & -a_{13}\left(y_{1}+x_{1}^{*}\right) & -a_{14}\left(y_{1}+x_{1}^{*}\right) \\
-a_{21}\left(y_{2}+x_{2}^{*}\right) & -a_{22}\left(y_{2}+x_{2}^{*}\right) & -a_{23}\left(y_{2}+x_{2}^{*}\right) & -a_{24}\left(y_{2}+x_{2}^{*}\right) \\
a_{31}\left(y_{3}+x_{3}^{*}\right) & a_{32}\left(y_{3}+x_{3}^{*}\right) & -r_{3}+a_{31} x_{1}^{*}+a_{32} x_{2}^{*} & 0 \\
a_{41}\left(y_{4}+x_{4}^{*}\right) & a_{42}\left(y_{4}+x_{4}^{*}\right) & 0 & -r_{4}+a_{41} x_{1}^{*}+a_{42} x_{2}^{*}
\end{array}\right], \\
\mathbf{B}=\left[\begin{array}{cccc}
0 & 0 & 1 & 0 \\
0 & 0 & 0 & 1
\end{array}\right]^{T},
\end{gathered}
$$

respectivamente. O ponto de equilíbrio é $\left(x_{1}^{*}, x_{2}^{*}, x_{3}^{*}, x_{4}^{*}\right)$. O equilíbrio do sistema pode ser recalculado, como mostrado em [10]. Assim, o ponto de equilíbrio escolhido, baseado no nível de pragas abaixo de danos econômicos, foi $(8 ; 10 ; 89,32 ; 8,88)$. As matrizes $\mathbf{Q}=\mathbf{C}^{T} \mathbf{C}$ e $\mathbf{C}=\operatorname{diag}\left(\sqrt{q_{i i}}\right)$.

A solução do problema do controle é dado pelas equações algébricas de Riccati independentes do estado, pois as matrizes de estado e do funcional são assumidas constantes a cada passo de integração. As resultados foram obtidos através do Matlab, onde as equações foram integradas pelo método Runge-Kutta de quarta ordem. As equações de Riccati foram resolvidas usando a função "LQR"do Matlab.

Foram escolhidos os valores das matrizes de peso $\mathbf{Q}=\operatorname{diag}(1,1,1,1)$ e $\mathbf{R}=$ $\operatorname{diag}(100,100)$. Um novo sistema de Riccati é resolvido a cada 0, 001 dias.

O resultado da simulação do sistema controlado está na Figura 2, com condição inicial $(30,40,20,5)$.

É possível observar na Figura 2 que o sistema estabiliza após um certo tempo, sendo que a primeira espécie de presas converge mais lentamente ao equilíbrio. Devese ressaltar que existe uma certa dependência do tempo gasto para o equilíbrio e os valores das matrizes de peso $\mathbf{Q}$ e $\mathbf{R}$, porém esta discussão não será abordada neste artigo.

Para análise de estabilidade considera-se o sistema sem controle $\dot{\mathbf{x}}=f(x)$. A estabilidade deste sistema pode ser examinada em torno da origem através da linearização. Se for assumido que $f(x) \in C^{1}$ e que o equilíbrio de interesse é a origem, então é possível verificar a estabilidade local assintótica através da aproximação linear de $f(x)$ em $x=0$. 

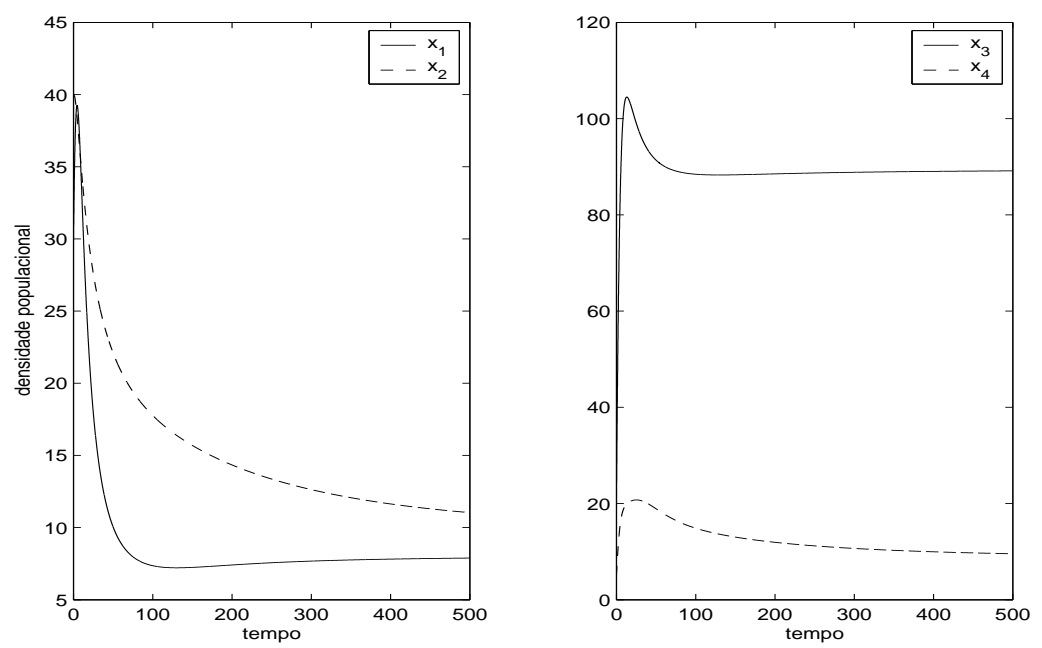

Figura 2: Trajetórias do sistema controlado, duas presas e dois predadores

\section{Conclusões}

O trabalho teve por objetivo principal a formulação e resolução do problema do controle ótimo do sistema não-linear presa-predador de Lotka-Volterra, através da metodologia SDRE e com garantia de estabilidade local. No modelo utilizado foi preservada a não-linearidade das equações, já que durante o processo de linearização alguns termos são desconsiderados, podendo exercer influência na dinâmica do sistema. As funções de controle também entraram no modelo de forma não-linear, porém, localmente, o controle foi linear.

A técnica de controle ótimo, proposta neste trabalho, para o controle de sistemas não-lineares, pode ser facilmente usada em sistemas não-lineares que aparecem em outras áreas do conhecimento. Numa situação real pode acontecer o regime descrito pelo modelo Lotka-Volterra, desde que existem fatores exógenos que asseguram certos valores dos coeficientes do modelo.

É importante a aplicação desses modelos em situações reais, o que muitas vezes não é fácil de conseguir, são muitos fatores que interferem numa situação real. $\mathrm{Na}$ modelagem matemática, geralmente é necessário dispensar alguns desses fatores para obter um modelo mais simples. Na aplicação real apresentada percebeu-se a necessidade do uso de controle, pois o sistema estava naturalmente equilibrado numa densidade de pragas bem acima do considerado abaixo de danos econômicos. A técnica de controle utilizada para o modelo real apresentado, nesse trabalho, mostrou-se eficaz, levando o nível de pragas abaixo de danos econômicos e não permitindo a extinção das espécies consideradas. 
Abstract. The purpose of this work is to choose optimal control strategies of the biological system which exhibits the nonlinear behavior. The control, based on two preys - one predator Lotka-Volterra model, is applied in soybean crop agroecosystems. The goal of this pest control strategy is to maintain the pest population density at level below the economic injury level. This optimal control problem is solved by State Dependent Riccati Equation (SDRE) method. Numerical simulations for proposed strategies of biological pest control based on the Lotka-Volterra model are provided to show the effectiveness of this method.

\section{Referências}

[1] A.O. Angulo, G.T.H. Weigert, Rachiplusia nu (Guenée), estados inmaduros y biología (Lepidoptera, Noctuidae), Bol. Soc. Biol. de Concepcion, Concepcion, Chile, (1974), 22-117.

[2] H.T. Banks, B.M. Lewis, H.T. Tran, Nonlinear feedback controllers and compensators: a State-Dependent Riccati Equation approach, Comput. Optim. Appl., 37 (2007), 177-218.

[3] H.T. Banks, Hee-Dae Kwon, J.A. Toivanen, H.T. Tran, A State-Dependent Riccati Equation - based estimator approach for HIV feedback control, Optimal Control Applications and Methods, 27 (2006), 93-121.

[4] R. Bellman, "Dynamic Programing", Princeton, New Jersey, 1957.

[5] S.R.B. Diehl, Soja: a importância do manejo de pragas, São Paulo: Correio Agrícola, 3 (1984), 660-665.

[6] M.E. Gilpin, Spiral chaos in a Predador-Prey model, American Naturalist, 113 (1979), 306-308.

[7] P.C. Mracek, J.R. Cloutier, Control designs for the nonlinear benchmark problem via the State-Dependent Riccati equation method, International Journal of robust and nonlinear control, 8 (1998), 401-433.

[8] A. Molter, M. Rafikov, Controle ótimo para um sistema caótico de LotkaVolterra, TEMA - Tendências em Matemática Aplicada e Computacional, 5, No. 2 (2004), 237-245.

[9] O. Nakono et al., "Etimologio Económica", Piracicaba: ESALQ, USP, 1981.

[10] M. Rafikov, J.M. Balthazar, H.F. von Bremen, Mathematical modeling and control of population systems: applications in biological pest control, Applied Mathematics and Computation, 200 (2008), 557-573.

[11] M. Rafikov, L. Bevilacqua, A.P.P. Wyse, Optimal control strategy of malaria vector using genetically modified mosquitoes, Journal of Theoretical Biology, 258 (2009), 418-425. 
[12] A.M. Shawky, A. W. Ordys, L. Petropoulakis, M.J. Grimble, Position control of flexible manipulator using non-linear $H_{\infty}$ with State-Dependent Riccati Equation, em "IMechE, 221 Part I: J. Systems and Control Engineering", pp. 475-486, 2007.

[13] R.R. Vence, Predation and Resource Partitioning in one Predator - two Prey, Model Community, American Naturalist, 112 (1978), 797-813, An Evolution, 13 (1998), 325-329.

[14] V. Volterra, "Leçons sur la Théorie Mathematique de la Lutte pour la Vie", Gauthier-Villars, Paris, 1931. 